\title{
Increased periostin expression affects the proliferation, collagen synthesis, migration and invasion of keloid fibroblasts under hypoxic conditions
}

\author{
ZHE ZHANG ${ }^{*}$, FANGFEI NIE* , CHUNFU KANG, BIN CHEN, ZELIAN QIN, \\ JIANXUN MA, YONGGUANG MA and XIA ZHAO
}

Department of Plastic Surgery, Peking University Third Hospital, Haidian, Beijing 100191, P.R. China

Received December 10, 2013; Accepted April 10, 2014

DOI: $10.3892 /$ ijmm.2014.1760

\begin{abstract}
Periostin, a secreted extracellular matrix protein, is involved in the wound healing and pathological process of various human cancers. Keloid scars are fibroproliferative tumor-like lesions and develop under local hypoxia. Using suppression subtractive hybridization, in a previous study, we found that periostin is overexpressed in keloids compared with hypertrophic scars. However, little is known about the regulation and function of periostin in keloids. In this study, we examined the effects of periostin on the bioactivity of keloid fibroblasts (KFs) in order to determine whether periostin is involved in hypoxia-stimulated keloid pathogenesis by measuring the expression levels of periostin in KFs cultured under hypoxic conditions. We also investigated the association between periostin and hypoxia-inducible factor-1 $\alpha$ (HIF-1 $\alpha)$. The mRNA, intracellular protein and secreted protein level of periostin was examined by RT-PCR (and quantitative PCR), western blot analysis and enzyme-linked immunosorbent assay (ELISA), respectively. We also used shRNA targeting periostin to knockdown its expression in the KFs. We report that hypoxia $\left(2 \% \mathrm{O}_{2}\right)$ upregulates both HIF- $1 \alpha$ and periostin expression in KFs. In addition, hypoxia-upregulated periostin expression was regulated by HIF-1 $\alpha$. The inhibition of periostin by short hairpin RNA decreased the hypoxia-stimulated proliferation, collagen synthesis, migration and invasion of KFs and altered the cell cycle, but did not affect apoptosis; treatment with recombinant human periostin protein reversed these effects. Periostin also activated the $\alpha v \beta 3$ integrin-PI3K/Akt pathway in the KFs. These findings suggest that hypoxia initiates hyperplasia of KFs and increases periostin expression under hypoxic
\end{abstract}

Correspondence to: Professor Zelian Qin, Department of Plastic Surgery, Peking University Third Hospital, 49 North Garden Road, Haidian, Beijing 100191, P.R. China

E-mail: qinzl@bjmu.edu.cn

*Contributed equally

Key words: periostin, hypoxia, fibroblast, keloid, hypoxia-inducible factor conditions; periostin is involved in the pathogenesis of keloids, which indicates that periostin may be a novel therapeutic target for keloids and other fibroproliferative disorders.

\section{Introduction}

Keloids, a pathological response to cutaneous wound healing, are characterized by the abnormal proliferation of fibroblasts and the excessive deposition of collagen (1). Clinically, keloids represent a thick tumor-like scar tissue that outgrows the original wound edges, invades the adjacent normal derma and rarely regresses over time (2). The effectiveness of the numerous treatments for keloids, including excision and intralesional corticosteroid injections, is still not satisfactory due to their high recurrence rate $(2,3)$.

Accumulating evidence suggests that a hypoxic microenvironment is associated with keloids due to an abnormally large number of occluded microvessels and that hypoxia plays a crucial role in keloid pathogenesis $(4,5)$. Hypoxia has been found to increase the expression of vascular endothelial growth factor (VEGF) in keloid fibroblasts (KFs) (6). The level of hypoxia-inducible factor- $1 \alpha(\mathrm{HIF}-1 \alpha)$ is consistently higher in freshly biopsied keloid tissues than their associated normal skin borders, which provides direct evidence of a local hypoxic state in keloids (7). HIF-1, the major transcription factor in response to hypoxia, is a heterodimeric molecule of 2 subunits, HIF-1 $\alpha$ and HIF-1 $\beta$. HIF-1 $\alpha$ has an oxygen-dependent degradation domain and its expression and activity are regulated by the cellular oxygen concentration, while HIF- $1 \beta$ is constitutively expressed. HIF-1 $\alpha$ binds to DNA on hypoxia response elements (HREs) in promoters of more than 60 target genes, such as VEGF and matrix metalloproteinases (MMPs), many involved in keloid formation $(8,9)$.

Periostin, a secreted extracellular matrix (ECM) protein, was initially identified in MC3TC-E1 mouse osteoblasts as a putative bone adhesion molecule (10). It was found to be upregulated in cutaneous wound healing, cutaneous fibrosis and tumor progression and is involved in cell survival, differentiation, metastasis and ECM remodeling $(11,12)$. Previously, we used suppression subtractive hybridization and found the upregulated expression of periostin among genes differentially expressed between keloids and hypertrophic scars (13). The 
expression of periostin is increased in keloids as compared with normal skin and may influence fibroblast proliferation (14).

Based on the above data, we hypothesized that periostin may play an important role in hypoxia-stimulated keloid pathogenesis. In the present study, we examined this hypothesis by examining the expression of periostin under hypoxic conditions $\left(2 \% \mathrm{O}_{2}\right)$, as well as the association between periostin and HIF-1 $\alpha$. Furthermore, we investigated the effects of periostin on hypoxia-stimulated KF bioactivity in terms of proliferation, cell cycle, apoptosis, collagen synthesis, migration and invasion; in addition, we aimed to identify the regulatory pathways involved.

\section{Materials and methods}

All experiments in this study were performed in compliance with the regulations of the Medical Ethics Committee of Peking University Third Hospital, Beijing, China.

Primary cell culture and treatment. KFs were isolated from discarded keloid tissues of patients who were undergoing surgery $(n=6)$. The characteristics of the study subjects are shown in Table I. All keloid tissue originated from untreated primary lesions. The KFs were maintained in Dulbecco's modified Eagle's medium (DMEM; Gibco, Carlsbad, CA, USA) supplemented with $10 \%$ fetal bovine serum (FBS; HyClone, Logan, UT, USA) containing $100 \mathrm{U} / \mathrm{ml}$ penicillin and $100 \mathrm{U} / \mathrm{ml}$ streptomycin (Invitrogen, Carlsbad, CA, USA). KFs at passages 4-8 were used for the following experiments. For hypoxic exposure, fibroblasts were placed in a modulator incubator in an atmosphere of $93 \% \mathrm{~N}_{2}, 5 \% \mathrm{CO}_{2}$ and $2 \% \mathrm{O}_{2}$. Normoxic conditions were defined as $20 \% \mathrm{O}_{2}$. Recombinant human periostin (rhPN; BioVendor, Brno, Czech) and echinomycin (Calbiochem, La Jolla, CA, USA) were applied at $10 \mathrm{ng} / \mathrm{ml}$ and $10 \mathrm{nM}$, respectively. For inhibition experiments, the cells were pre-treated with $30 \mu \mathrm{M}$ LY294002 (inhibitor of PI3K; Cell Signaling Technology, Beverly, MA, USA) or $20 \mu \mathrm{g} / \mathrm{ml}$ each of $\alpha \mathrm{v} \beta 3$ or $\alpha \mathrm{v} \beta 5$-integrin antibody (Millipore, Billerica, MA, USA) for $1 \mathrm{~h}$ at $37^{\circ} \mathrm{C}$.

Reverse transcription (RT)-polymerase chain reaction $(P C R)$ and quantitative PCR. Total RNA was extracted using TRIzol reagent (Tiangen, Beijing, China), and cDNA was acquired using reverse transcriptase (Thermo Scientific, Waltham, MA, USA). Quantitative PCR was carried out using the Maxima SYBR-Green I qPCR Master Mix (Fermentas, Waltham, MA, USA) with a qPCR/Real-Time PCR Instrument (Bio-Rad, Hercules, CA, USA). cDNA was amplified with the primer sequences for periostin (forward, 5'-tcattggaaaaggatttgaacc-3' and reverse, 5'-caggtgtgtctgctggatagag-3'; 189 bp); collagen I (forward, 5'-ttctgtacgcaggtgattgg-3' and reverse, 5'-catgttcag ctttgtggacc-3'; $129 \mathrm{bp}$ ) and $\beta$-actin (forward, 5'-agcgagcat ccccaaagtt-3' and reverse, 5'-gggcacgaaggctcatcatt-3'; 285 bp). For quantification, target gene expression was normalized to that of $\beta$-actin in each sample. Data analysis was carried out using the $\Delta \Delta \mathrm{Ct}$ method.

Western blot analysis. The KFs were washed twice with icecold phosphate-buffered saline (PBS), then harvested with lysis buffer containing phosphatase and protease inhibitors. The protein concentration was quantified using the BCA Protein
Table I. Characteristics of patients enrolled in this study.

\begin{tabular}{lcllc}
\hline Patient & $\begin{array}{c}\text { Age } \\
\text { (years) }\end{array}$ & Gender & Biopsy site & $\begin{array}{c}\text { Duration } \\
\text { (years) }\end{array}$ \\
\hline K1 & 24 & Female & Chest & 2 \\
K2 & 33 & Female & Earlobe & 2 \\
K3 & 50 & Male & Earlobe & 4 \\
K4 & 25 & Female & Chest & 1 \\
K5 & 34 & Female & Chest & 1 \\
K6 & 8 & Male & Instep & 0.5 \\
\hline
\end{tabular}

Assay kit (CWbiotech, Beijing, China). Proteins in lysates were separated by SDS-PAGE, then transferred onto nitrocellulose membranes (Applygen Technologies, Inc., Beijing, China), and incubated with primary antibodies to rabbit anti-periostin (1:1,000 dilution), rabbit anti-HIF1 $\alpha$ (1:500 dilution), rabbit anti-collagen I (1:1,000 dilution), rabbit anti-Akt (1:1,000 dilution) or anti-phospho-Akt (1:1,000 dilution) (all from Abcam, Cambridge, MA, USA), or mouse anti- $\beta$-actin (ZsBio, Beijing, China), then corresponding IgG secondary antibodies (1:10,000 dilution; LI-COR Biosciences, Lincoln, NE, USA). The membranes were scanned using the Odyssey Infrared Imaging System (LI-COR Biosciences).

ELISA. Secreted periostin was measured with use of an ELISA kit (R\&D Systems, Minneapolis, MN, USA). Briefly, 96-well microplates were coated overnight with capture antibody, washed 3 times with wash buffer, then blocked with reagent diluent for $1 \mathrm{~h}$. An amount of $100 \mu \mathrm{l}$ of all standards and cell medium samples was added to the 96-well plate for incubation for $2 \mathrm{~h}$. After $2 \mathrm{~h}$ of incubation with detection antibody, a 20-min incubation with a working dilution of horseradish peroxidaseconjugated streptavidin and a 20-min incubation with substrate solution in a light-resistant container, stop solution was added to each well. The absorbance of periostin was calculated by measuring the absorbance at $450 \mathrm{~nm}$, correcting for plate artifact at $570 \mathrm{~nm}$ and utilizing a log-transformed standard curve.

Lentiviral-mediated stable gene knockdown in KFs. Short hairpin RNA (shRNA) was prepared and a vector-based shRNA plasmid was constructed. Briefly, one 21-nt siRNA duplex (aaactgaaggacccacactaa) against the human periostin consensus coding sequence (GenBank accession no. NM006475) or one non-silencing luciferase sequence was inserted into an shRNA oligonucleotide template, which was subcloned into the pGCsi shRNA expression vector encoding green fluorescent protein (GFP; GeneChem, Shanghai, China). The lentivirus harboring shRNA periostin or non-silencing shRNA and the GFP gene were transfected into the cells in serum-free DMEM for $10 \mathrm{~h}$. The medium was then replaced with DMEM and 10\% FBS. The GFP-positive cells were separated by flow cytometry. The KFs were divided into 3 groups: cells transfected with PBS as a normal control, non-silencing shRNA as a negative control (NC group), or shRNA periostin (sh-pn group). Periostin mRNA and intracellular protein levels were reduced by 64 and $60 \%$, respectively, as compared with the NC cells, with no difference between the normal and NC cells. 
A

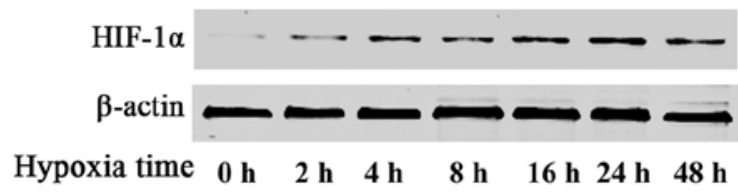

C
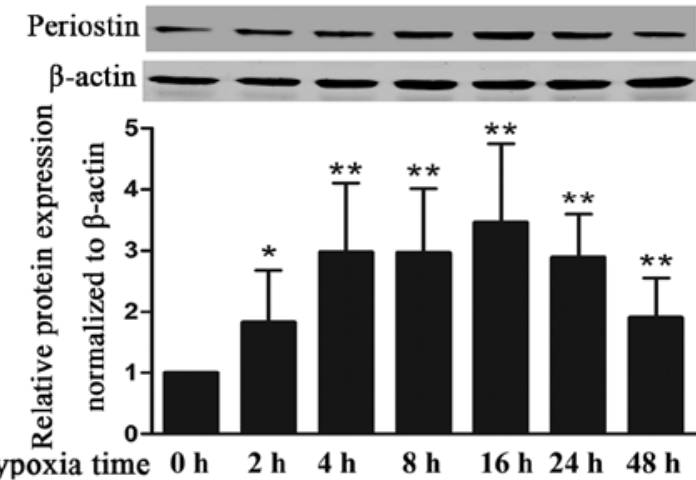

B

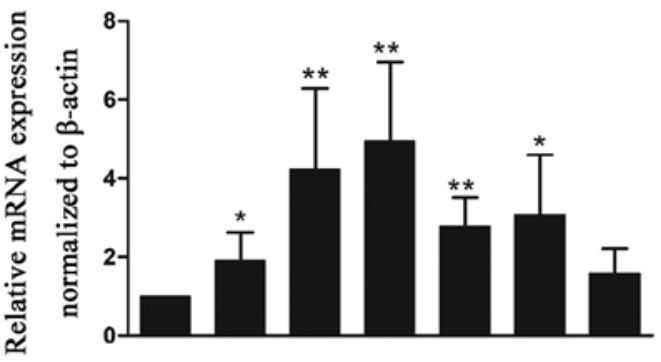

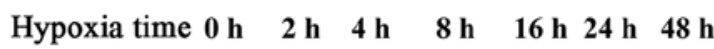

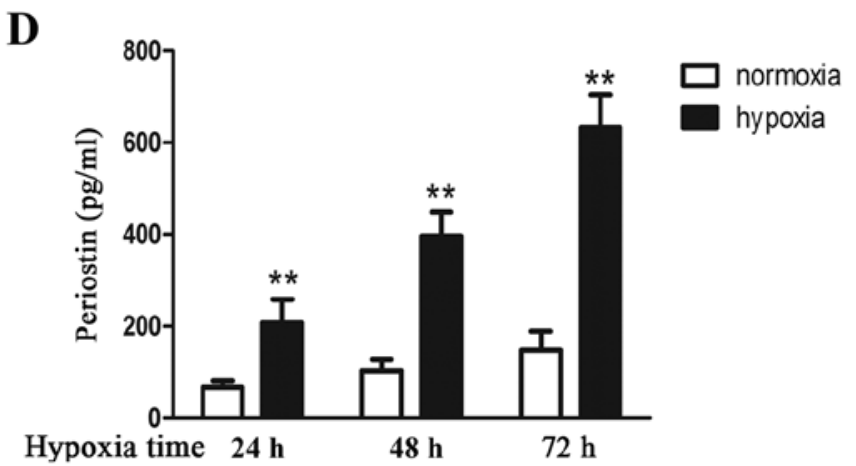

Figure 1. Periostin and hypoxia-inducible factor-1 $\alpha$ (HIF-1 $\alpha)$ expression was increased under hypoxic conditions in a time-dependent manner. Keloid fibroblasts (KFs) were exposed to hypoxia $\left(2 \% \mathrm{O}_{2}\right)$ for various periods of time or normoxia. (A) Western blot analysis of HIF-1 $\alpha$ protein level under hypoxic conditions. (B) RT-PCR analysis of the periostin mRNA level, and (C) western blot analysis of intracellular periostin protein level. (D) ELISA of secreted periostin protein level. Data are the means \pm standard deviation (SD) from at least 3 independent experiments. ${ }^{*} \mathrm{P}<0.05,{ }^{* *} \mathrm{P}<0.01$ vs. cells exposed to normoxia $(0 \mathrm{~h})$, $\mathrm{n}=6$.

Cell proliferation assay. Cells at $70 \%$ confluence were serumstarved in $0.1 \%$ FBS for $24 \mathrm{~h}$ for synchronization, then plated at $5 \times 10^{3}$ cells/well in 96-well plates under hypoxic conditions. The cells were incubated with fresh DMEM containing 10\% FBS. The cell counting kit-8 (CCK-8; Dojindo, Kumamoto, Japan) was used to determine KF viability at various time points. For CCK- 8 assay, the cells were incubated with $10 \mu \mathrm{l}$ CCK-8 solution for $1 \mathrm{~h}$ and the absorbance was measured at $450 \mathrm{~nm}$.

Flow cytometry. For cell cycle analysis, KFs transfected with or without lentivirus were cultured in $60-\mathrm{mm}$ dishes under hypoxic conditions for $48 \mathrm{~h}$, then harvested in PBS. The cell suspension was stained with propidium iodide (PI; eBioscience, San Diego, CA, USA) and the cell cycle was analyzed using the FACSCalibur system (BD Biosciences, San Jose, CA, USA). Apoptosis was measured using an Annexin V apoptosis detection kit with APC (eBioscience). The cells were serumstarved for $24 \mathrm{~h}$, then complete medium was added. Following exposure to hypoxia, the cells stained with APC-conjugated Annexin V and PI were pelleted and analyzed according to the manufacturer's instructions, and data were analyzed using FlowJo software (Tree Star, Inc., Ashland, OR, USA).

Migration and invasion assay. Migration and invasion in vitro were measured in a Transwell chamber. An amount of $100 \mu 1$ Matrigel (BD Biosciences) was coated onto the upper chambers of the Transwell inserts ( $6.5 \mathrm{~mm}, 8 \mu \mathrm{m}$ pore size; Millipore) for the invasion assay but not for the migration assay. Following pre-incubation with serum-free medium for $12 \mathrm{~h}, 1 \times 10^{4}$ cells (migration assay) or $5 \times 10^{4}$ cells (invasion assay) per well were seeded for $24 \mathrm{~h}$ in $0.1 \%$ FBS medium into the upper chamber, and the lower chamber contained 10\% FBS medium. The cells were fixed in paraformaldehyde for $15 \mathrm{~min}$, and the cells at the bottom of the membrane were stained with crystal violet for $30 \mathrm{~min}$. Cells in migration and invasion assay stained with crystal violet from 6 randomly selected fields were counted under a microscope (x100 magnification) and the mean for each chamber was determined.

Statistical analysis. Statistical analysis was carried out using SPSS 12.0 software (SPSS Inc., Chicago, IL, USA). Each experiment was performed in triplicate. Quantitative data are presented as the means \pm standard deviation (SD). One-way ANOVA followed by Scheffe's post-hoc test were used to compare multiple groups. Values of $\mathrm{P}<0.05$ were considered to indicate statistically significant differences.

\section{Results}

Increased expression of periostin in KFs under hypoxic conditions. To examine the effects of hypoxia on the expression of periostin in KFs, we cultured KFs under hypoxic conditions and measured the HIF-1 $\alpha$ and periostin levels. As expected, exposure to hypoxia markedly increased the protein expression of HIF-1 $\alpha$, which indicates that KFs exist within a hypoxic environment (Fig. 1A). Hypoxia also increased the expression of periostin; mRNA level (4.9-fold increase) and the intracellular protein level (3.1-fold increase) peaked at 8 and $16 \mathrm{~h}$, respectively (Fig. 1B and C). Periostin mRNA and intracellular protein levels were maintained at relatively high levels even with $48 \mathrm{~h}$ of exposure to hypoxia. The secreted protein in the cell supernatant under hypoxic conditions was increased by 3-, 3.8- and 4.2-fold at 24, 48 and $72 \mathrm{~h}$ as compared with cells cultured under normoxic conditions (Fig. 1D). 

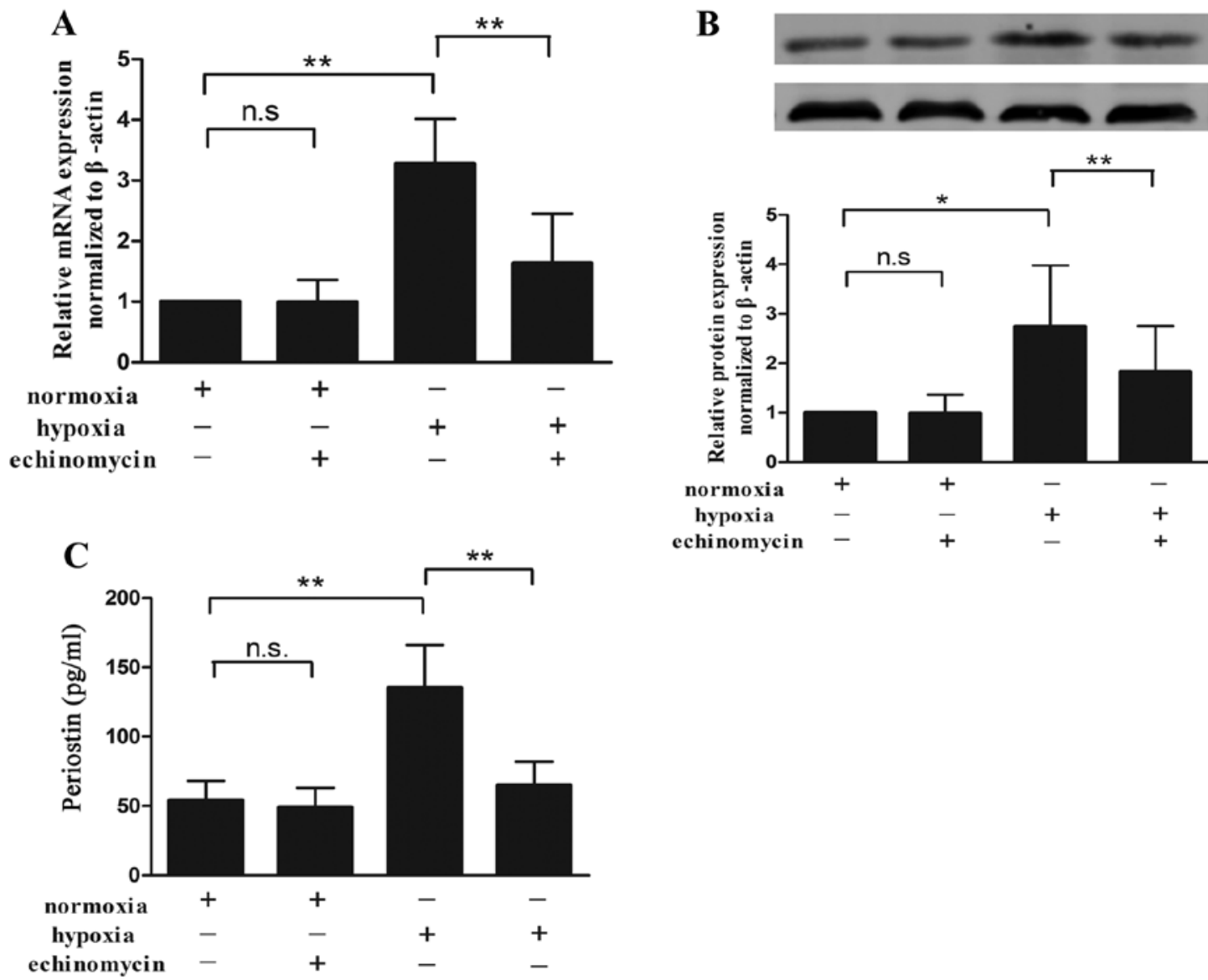

Figure 2. Inhibition of hypoxia-inducible factor-1 $\alpha$ (HIF-1 $\alpha$ ) activity reduces the expression of periostin in keloid fibroblasts (KFs) under hypoxic conditions KFs were exposed to normoxia or hypoxia, with or without echinomycin for $12 \mathrm{~h}$. Quantitative RT-PCR analysis of (A) periostin mRNA level, (B) western blot analysis of intracellular protein level and (C) ELISA of the secreted protein level. Data are the means \pm standard deviation (SD) from at least 3 independent experiments. ${ }^{*} \mathrm{P}<0.05,{ }^{* *} \mathrm{P}<0.01$. n.s., not significant, $\mathrm{n}=6$.

Upregulation of periostin expression induced by hypoxia is $H I F-1 \alpha$-dependent. To investigate the association between periostin and HIF-1 $\alpha$ in the KFs, we incubated cultured KFs under hypoxic or normoxic conditions with $10 \mathrm{nM}$ echinomycin, a small-molecule cyclic-peptide antibiotic known to specifically inhibit HIF transcriptional activity by suppressing its binding to the HRE site of target genes (15). The periostin mRNA level under normoxic conditions was not affected by echinomycin as compared with the controls (no echinomycin). Hypoxia enhanced the periostin mRNA expression, and echinomycin abolished this effect (Fig. 2A). The levels of intracellular protein (Fig. 2B) and secreted protein in the cell supernatant (Fig. 2C) showed a similar effect.

Effects of periostin on hypoxia-stimulated KF proliferation, cell cycle distribution and apoptosis. Hypoxia increased the proliferation of KFs by 26, 19 and $29 \%$ at 24, 48 and $72 \mathrm{~h}$, respectively (Fig. 3A). To further examine the effects of periostin on KF proliferation, we transfected the KFs with shRNA against periostin or control shRNA. Fluorescence microscopy, quantitative PCR and western blot analyses were performed to ensure the efficiency of transfection before each experiment (Fig. 4). The knockdown of periostin significantly decreased cell proliferation by 13,11 and $16 \%$ at 24,48 and $72 \mathrm{~h}$, respectively, which was reversed by incubation with
rhPN (Fig. 3A). The KFs cultured under hypoxic conditions showed an increased number of cells in the $\mathrm{G} 2 / \mathrm{M}$ phase and a decreased number of cells in the G0/G1 phase (Fig. 3B). The knockdown of periostin by shRNA resulted in G1/S cell cycle arrest, which was reversed by rhPN treatment. We also evaluated the anti-apoptotic role of periostin in KFs by Annexin V treatment (Fig. 3C). Neither hypoxia nor the knockdown of periostin affected the apoptosis of KFs.

Effects of periostin on hypoxia-stimulated collagen synthesis, migration and invasion of KFs. Keloids exhibit aberrant, exuberant collagen synthesis and deposition (16). Type I collagen at both the mRNA (Fig. 5A) and protein (Fig. 5B) level was increased in the KFs cultured under hypoxic conditions as compared with those cultured under normoxic conditions. The inhibition of periostin by shRNA decreased the expression of type I collagen, and this effect was reversed by exogenous rhPN treatment.

The notable migratory activity of KFs and the invasive nature of keloids are a hallmark in keloids (17). In our study, we found that a greater number of KFs migrated to and invaded the lower compartment of Transwell chambers under hypoxic conditions as compared with normoxic conditions (Fig. 6). The knockdown of periostin decreased the number of KFs migrating and invading, which was reversed by rhPN treatment. 
$\mathbf{A}$

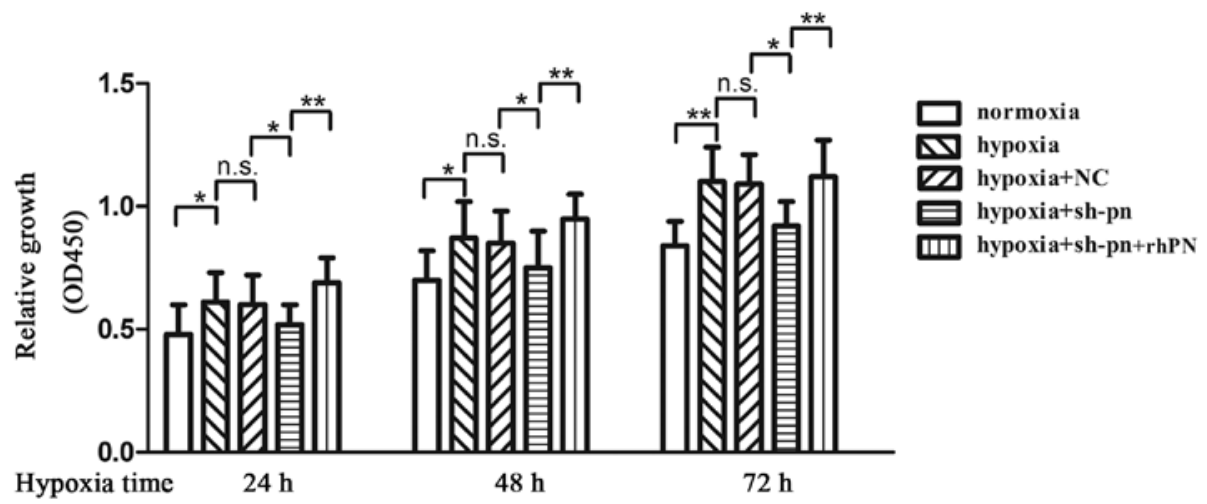

B

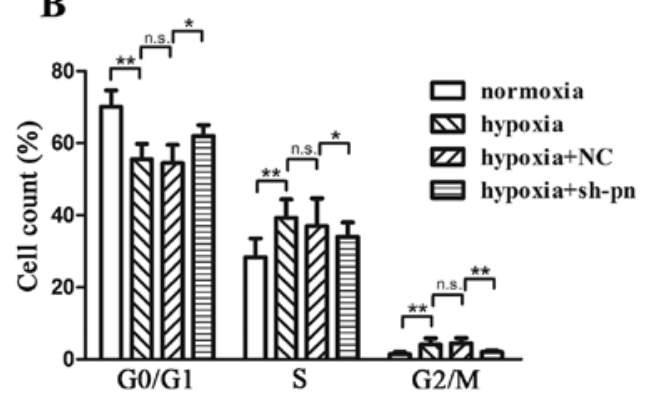

C

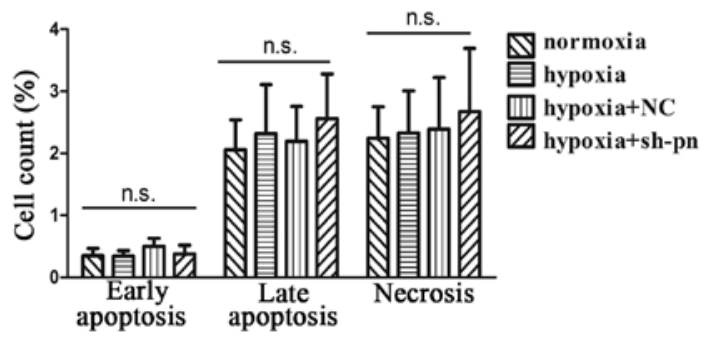

Figure 3. Effect of periostin on proliferation, cell cycle and apoptosis of keloid fibroblasts (KFs) under hypoxic conditions. (A) Proliferation of KFs cultured under normoxic or hypoxic conditions over various periods of time and following transfection with short hairpin RNA (shRNA) against periostin (sh-pn) or treatment with recombinant human periostin (rhPN; $10 \mathrm{ng} / \mathrm{ml}$ ). (B) Cell cycle of KFs cultured under hypoxic conditions and following treatment with shRNA against periostin. (C) Number of apoptotic and necrotic cells following exposure to hypoxia and knockdown of periostin. Data are the means \pm standard deviation (SD) from at least 3 independent experiments. $\mathrm{P}<0.05$. n.s., not significant, $\mathrm{n}=6$.
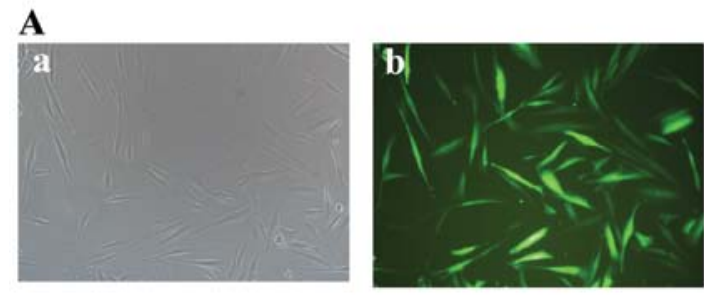

B

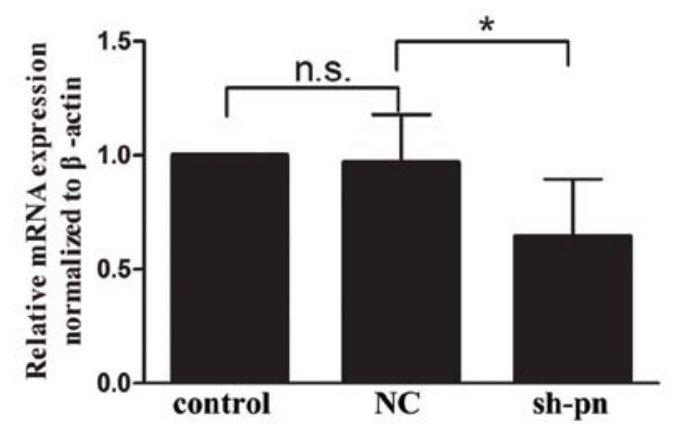

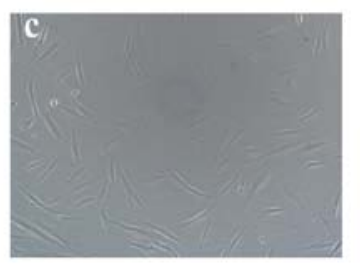

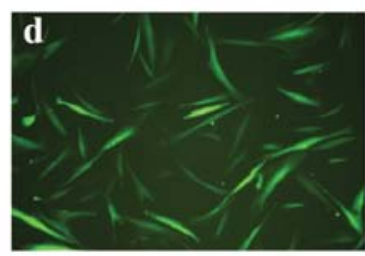

C

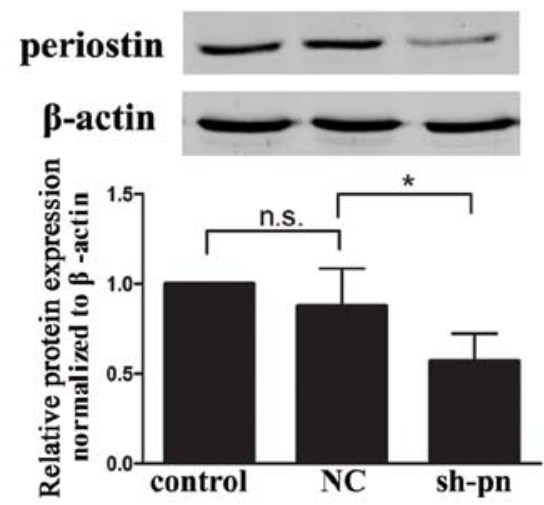

Figure 4. Lentiviral-mediated stable genetic knockdown of periostin in keloid fibroblasts (KFs). KFs were transfected with lentivirus harboring short hairpin RNA (shRNA) against periostin (sh-pn) or non-silencing shRNA [negative control (NC)]. (A) Fluorescence microscopy, (B) quantitative PCR and (C) western blot analyses were performed to ensure the efficiency of transfection before each experiment. (A) shRNA-periostin (sh-pn) under (a) microscopy and (b) fluorescence microscopy; non-silenced group under (c) microscopy and (d) fluorescence microscopy. Transfection efficiency symbolized by KFs expressing green fluorescent protein (GFP) was approximately $90 \%$. Data are the means \pm standard deviation (SD) from at least 3 independent experiments. ${ }^{*} \mathrm{P}<0.05$. n.s., not significant, $\mathrm{n}=6$.

Role of integrin-Akt pathway in periostin-induced proliferation of KFs. The PI3K/Akt pathway is one of most potent proliferative signaling pathways in human fibroblasts and is involved in keloid formation (18). In our study, we found that hypoxia increased 
$\mathbf{A}$

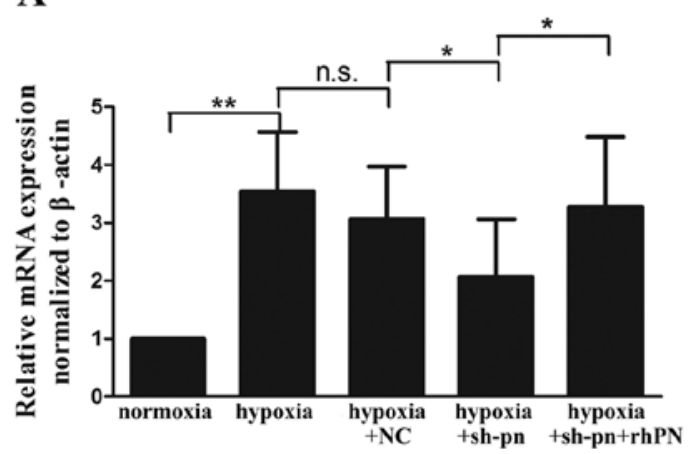

B

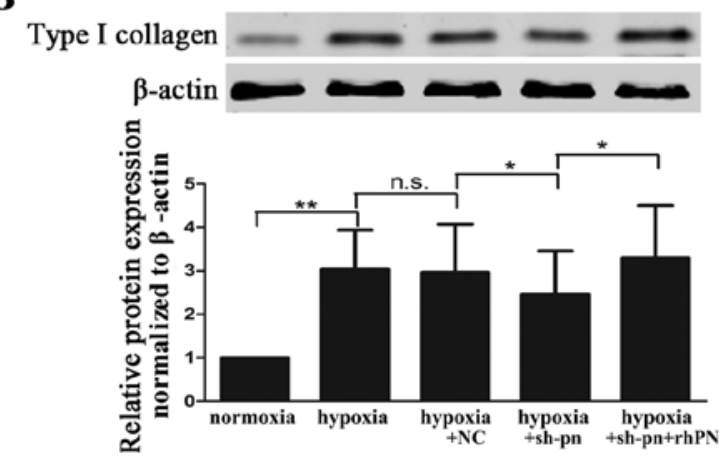

Figure 5. Periostin increases collagen synthesis in keloid fibroblasts (KFs) under hypoxic conditions. The expression of type I collagen in KFs cultured under hypoxic conditions for $24 \mathrm{~h}$ or under normoxic conditions with or without periostin knockdown. (A) RT-PCR analysis of the periostin mRNA level, and (B) western blot analysis of the intracellular protein level. Data are the means \pm standard deviation (SD) from at least 3 independent experiments. ${ }^{*} \mathrm{P}<0.05$, ${ }^{* *} \mathrm{P}<0.01$. n.s., not significant, $\mathrm{n}=6$.
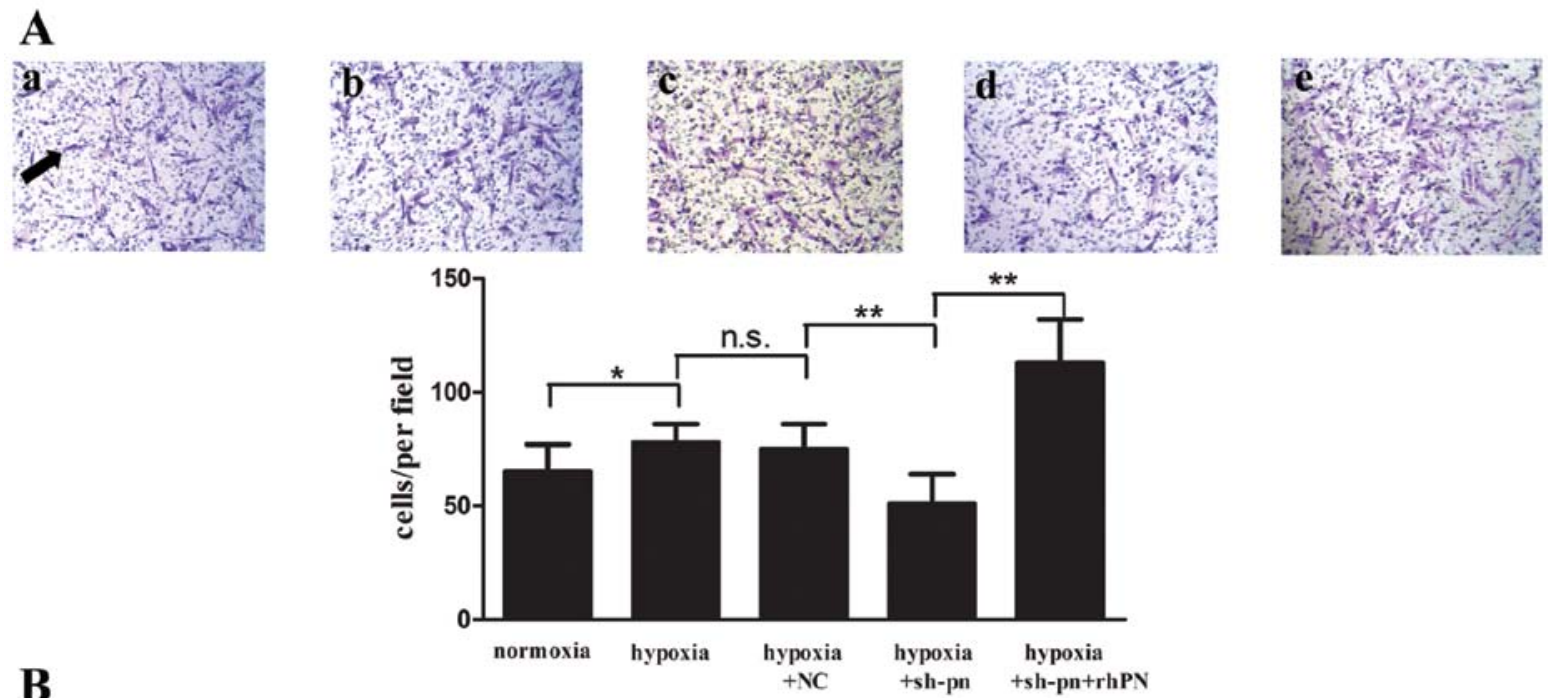

\section{B}
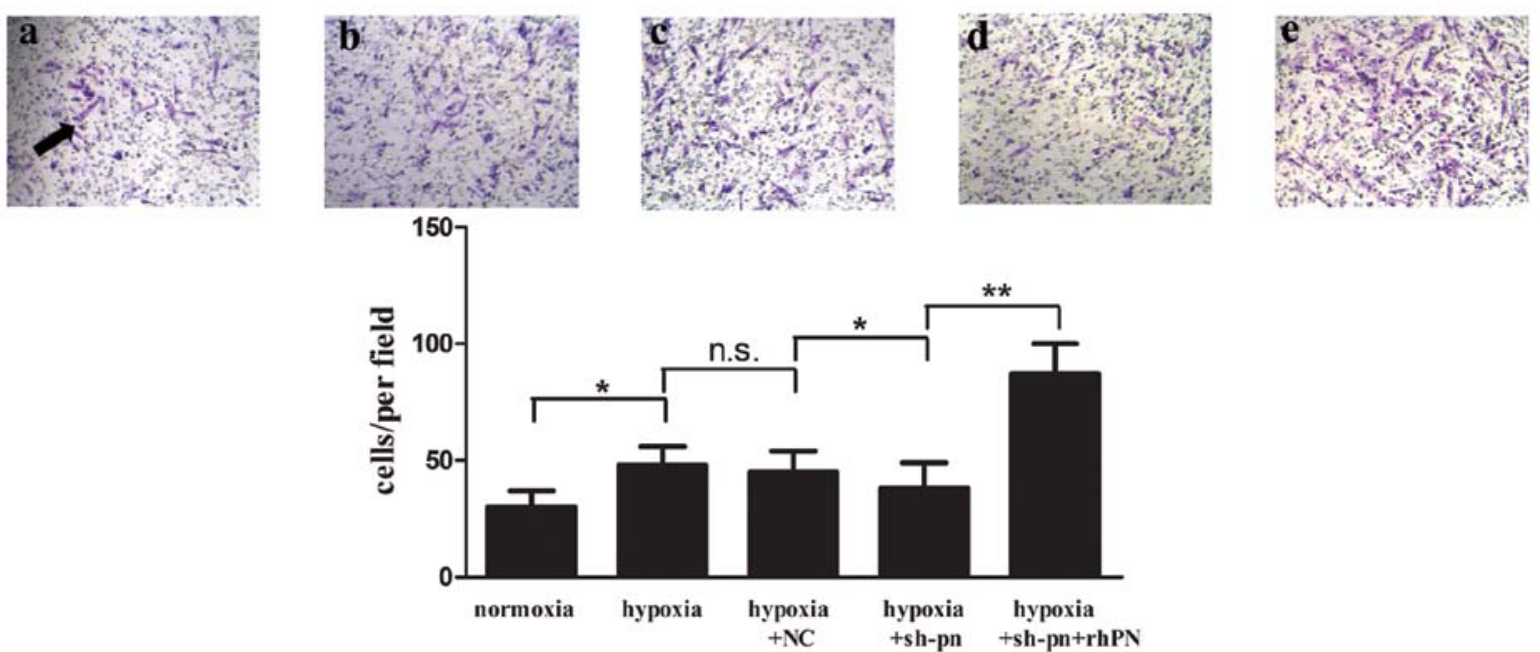

Figure 6. Effect of periostin on the migration and invasion of keloid fibroblasts (KFs) under hypoxic conditions. The mean number of (A) migrating or (B) invading cells (arrow) counted in 6 randomly selected fields. KFs under (a) normoxic conditions, (b) hypoxic conditions, (c) transfected with non-silencing RNA [negative control (NC)] under hypoxic conditions, (d) transfected with short hairpin RNA (shRNA) targeting periostin (sh-pn) under hypoxic conditions, (e) KFs transfected with sh-pn and treated with recombinant human periostin (rhPN) under hypoxic conditions. Data are the means \pm standard deviation (SD) from at least 3 independent experiments. ${ }^{*} \mathrm{P}<0.05,{ }^{* * *} \mathrm{P}<0.01$. n.s., not significant, $\mathrm{n}=6$.

the level of phosphorylated Akt, and the phosphorylated Akt level was lower in the KFs transfected with shRNA targeting periostin than in the normal and negattive control-transfected (non-silenced) KFs under hypoxic conditions (Fig. 7A). Akt phosphorylation was increased 5 min following rhPN stimulation, with a maximal increase observed at $240 \mathrm{~min}$, and then 
A

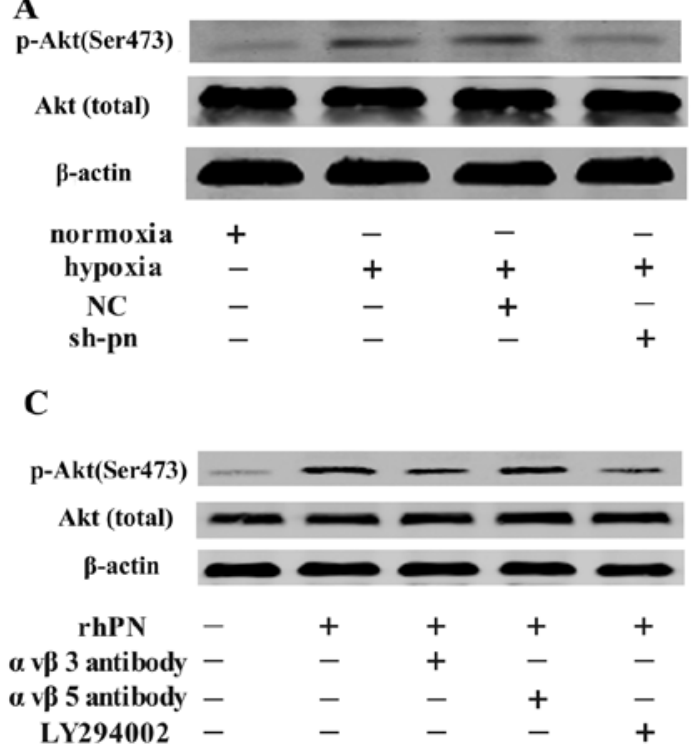

B
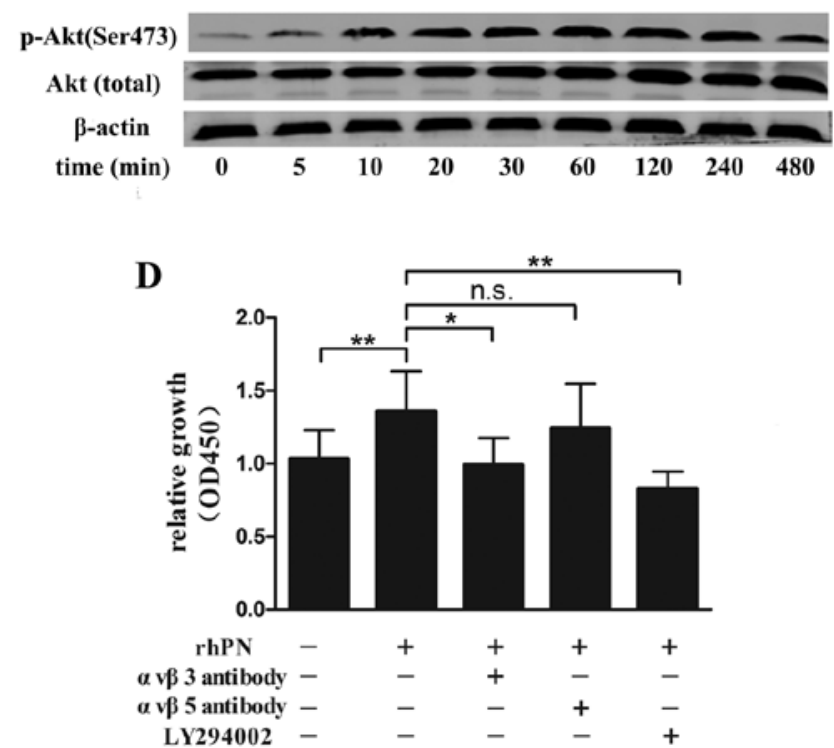

Figure 7. Periostin activates the $\alpha v \beta 3$ integrin-PI3K/Akt signaling pathway in keloid fibroblasts (KFs). (A) Western blot analysis of Akt phosphorylation in KFs cultured under hypoxic conditions and transfected with short hairpin RNA (shRNA) against periostin (sh-pn) and (B) in KFs treated with $10 \mathrm{ng} / \mathrm{ml}$ recombinant human periostin (rhPN) for the indicated periods of time. (C) KFs were incubated with $\alpha \mathrm{v} \beta 3$-integrin antibody, $\alpha \mathrm{v} \beta 5$-integrin antibody (both $20 \mu \mathrm{g} / \mathrm{ml}$ ) or PI3K inhibitor, LY294002 $(30 \mu \mathrm{M})$ for $1 \mathrm{~h}$, then treated with rhPN (10 ng/ml). Western blot analysis of (C) Akt phosphorylation and (D) CCK-8 analysis of KF proliferation. Data are the means \pm standard deviation (SD) from at least 3 independent experiments. ${ }^{*} \mathrm{P}<0.05,{ }^{* *} \mathrm{P}<0.01$. NC, negative control; $\mathrm{n} . \mathrm{s}$., not significant, $\mathrm{n}=6$.

decreased gradually for up to $12 \mathrm{~h}$ but remained at a higher level compared with the control (no rhPN stimulation; Fig. 7B). Periostin is a ligand for selected integrins (19); thus, we preincubated the KFs with $\alpha v \beta 3$ - or $\alpha v \beta 5$-integrin antibody or the PI3K inhibitor, LY294002, prior to exposure to hypoxia. The $\alpha v \beta 3$ antibody and LY294002 but not $\alpha v \beta 5$ antibody decreased Akt phosphorylation (Fig. 7C) and the proliferation of the KFs (Fig. 7D) as compared with the controls (untreated cells).

\section{Discussion}

Normal wound healing has stop signals to end the course of repair when the defective dermis is repaired and epithelialization is complete. When a microenvironment or genetic change results in futile or ineffective signals, the repair process continues and results in excessive scar tissue (20). Keloid scar tissue most frustrating to physicians due to the lack of ineffective therapy. Keloids, generally considered benign hyperplastic dermal tumors, exhibit abnormalities in cell proliferation, migration and invasion, they escape from apoptosis, and are disproportionately accumulated in the ECM (2). Keloids thrive in a hypoxic microenvironment, In this study, we reported that the periostin level was increased in KFs in response to a hypoxic environment, and that its expression was regulated by HIF-1 $\alpha$. In addition, the upregulated periostin expression promoted hypoxia-stimulated KF bioactivity in terms of cell proliferation, collagen synthesis, migration and invasion, and altered the cell cycle and activated the $\alpha v \beta 3$ integrin-PI3K/ Akt signaling pathway. The inhibition of periostin by shRNA decreased the hypoxia-stimulated KF bioactivity. Thus, periostin may be a target for the treatment of keloids.

A relative hypoxic state exists within the zone of injury in early-stage healing wounds and initiates wound repair by inducing fibroblast proliferation and angiogenesis (21).
Kisher (22) hypothesized that keloids, resulting from excessive repair, may be an aberration of the hypoxic microenvironment. The hypoxic microenvironment in keloids was confirmed by observing microvascular occlusion by a transmission electron microscope and HIF-1 $\alpha$ expression in keloid tissue (23). In this study, cultured KFs exposed to a hypoxic environment showed a marked time-dependent increase in both the mRNA and protein levels of periostin. The result was consistent with previous findings that hypoxic stress increases the expression of periostin in human periodontal ligament cells and rat pulmonary arterial smooth muscle cells $(24,25)$. Furthermore, we found that the HIF-1 $\alpha$ level was upregulated along with the periostin level and increased its expression. HIF-1 $\alpha$ regulates the expression of ECM-associated proteins. Deschene et al (26) showed that the secretion of collagen and MMP2 by fibroblasts was mediated by the transcriptional regulatory activity of HIF. In addition, HIF-1 $\alpha$ has been shown to increase the expression of plasminogen activator inhibitor 1 at both the transcriptional and post-transcriptional level in KFs (7). Thus, hypoxia may contribute to keloid formation by activating HIF-1 $\alpha$ and its downstream proteins, including periostin, to remodel the ECM.

Although keloids are a benign disorder, they invade the adjacent normal derma and KFs display a bioactivity similar to that of tumors (27). Hypoxia is thought to promote the growth and invasion of tumors (28). In this study, we found that hypoxia stimulated the proliferation, mobility and collagen production of KFs, which provides additional evidence of the involvement of hypoxia in keloid pathogenesis. Fibroblasts are responsible for the synthesis, deposition and remodeling of the ECM, and the release and activation of growth factors and cytokines by KFs are essential to keloid formation, in which the roles of VEGF, insulin-like growth factor and transforming growth factor- $\beta$ (TGF- $\beta$ ) have been identified $(6,29,30)$. TGF- $\beta$, 
the most potent profibrotic cytokine, increases the proliferation of and collagen synthesis in KFs (31). In this study, we found that the inhibition of periostin by shRNA decreased hypoxiastimulated cell proliferation, migration, invasion and collagen synthesis; these effects are similar to those of TGF- $\beta$ (32). Periostin has a similar structure as $\beta$ igH 3 , which also retains the 4 conserved fasciclin I domains and conserved motifs to bind to the integrin receptor family (33), and periostin is upregulated by TGF- $\beta 1$ in lung fibroblast cells (34). Thus, the association between periostin and TGF- $\beta$ requires further study.

Apoptosis mediates the transition from granulated tissue to scar atrophy (35). Periostin exerts an anti-apoptosis effect in many tumors under hypoxic conditions (36). However, in this study, we found that KF apoptosis showed no significant difference between the NC group and the sh-pn group under hypoxic conditions. We hypothesized that this is due to the following reasons: i) in general, only severe and prolonged hypoxia initiates apoptosis, whereas cells can adapt to and survive under acute and mild hypoxia (37); the hypoxic conditions used in our study $\left(2 \% \mathrm{O}_{2}\right)$ may not be sufficient to mimic the stressinduced hypoxic conditions of keloid formation in vivo; ii) we only partially decreased the level of periostin; the remaining periostin may prevent KFs from undergoing apoptosis; and iii) in another study, we found that various concentrations of periostin did not prevent the apoptosis of fibroblasts under serum-free conditions (unpublished data); thus periostin may have little effect on the regulation of apoptosis of KFs.

Periostin has been studied as a ligand for certain integrins, such as $\alpha v \beta 3$ and $\alpha v \beta 5$, and its downstream PI3K/Akt signaling pathway has been well documented to be involved in fibroblast cell proliferation, survival and migration (38). Our data showed that periostin can significantly promote the phosphorylation of Akt in KFs. Furthermore, $\alpha v \beta 3$ integrin antibody and LY294002, but not $\alpha v \beta 5$ antibody inhibited the periostin-stimulated Akt phosphorylation and proliferation of KFs. Our results suggest that the upregulation of periostin under hypoxic conditions affects the proliferation of KFs by activating the $\alpha v \beta 3$ integrin-PI3K/Akt signaling pathway.

Wound repair initiates a cascade of pathological events, including inflammation, tissue formation and tissue remodeling (39). Previously, we (40), as well as others (11) found that periostin was located in the granulation tissue beneath the extended epidermal wound edges and in the dermal-epidermal junctions and promoted cutaneous wound healing by enhancing the proliferation and migration of dermal fibroblasts. Periostin regulates myofibroblast differentiation and collagen matrix contraction, and periostin-deficient animal models exhibit delayed wound repair (41). In addition, it affects collagen production by fibroblasts following acute myocardial infarction (42). The normal regulation of periostin may be required throughout the repair process, and the dysregulation of periostin may tip the balance toward excessive repair, resulting in keloid formation and other fibroproliferative disorders. Our results suggest that a hypoxic microenvironment unduly prolongs the skin repair process due to the overexpression of certain factors, including HIF-1 $\alpha$ and periostin, which may contribute to keloid formation.

Thus, we conclude that hypoxia initiates KF hyperplasia, and that periostin may be a novel factor contributing to the abnormal biological behaviour of KFs under hypoxic conditions and to the progression of keloids. Our findings may contribute to a better understanding of keloid pathogenesis and the development of novel therapeutic approaches for keloids and other fibroproliferative disorders.

\section{Acknowledgements}

This study was supported by grants from the National Natural Science Foundation of China (no. 30973126) and the Ministry of Education doctoral foundation of the People's Republic of China (no. 20130001110095). We thank the teachers of the Medical Research Center of Peking University Third Hospital for their excellent technical assistance and Laura Smales for providing assistance with the language.

\section{References}

1. Niessen FB, Spauwen PH, Schalkwijk J and Kon M: On the nature of hypertrophic scars and keloids: a review. Plast Reconstr Surg 104: 1435-1458, 1999.

2. Bran GM, Goessler UR, Hormann K, Riedel F and Sadick H: Keloids: Current concepts of pathogenesis (Review). Int J Mol Med 24: 283-293, 2009.

3. Love PB and Kundu RV: Keloids: an update on medical and surgical treatments. J Drugs Dermatol 12: 403-409, 2013.

4. Zhang Q, Wu Y, Ann DK, et al: Mechanisms of hypoxic regulation of plasminogen activator inhibitor-1 gene expression in keloid fibroblasts. J Invest Dermatol 121: 1005-1012, 2003.

5. Ueda K, Yasuda Y, Furuya E and Oba S: Inadequate blood supply persists in keloids. Scand J Plast Reconstr Surg Hand Surg 38: 267-271, 2004.

6. Steinbrech DS, Mehrara BJ, Chau D, et al: Hypoxia upregulates VEGF production in keloid fibroblasts. Ann Plast Surg 42: 514-520, 1999.

7. Zhang Q, Wu Y, Chau CH, Ann DK, Bertolami CN and Le AD: Crosstalk of hypoxia-mediated signaling pathways in upregulating plasminogen activator inhibitor-1 expression in keloid fibroblasts. J Cell Physiol 199: 89-97, 2004.

8. Ahluwalia A and Tarnawski AS: Critical role of hypoxia sensor - HIF-lalpha in VEGF gene activation. Implications for angiogenesis and tissue injury healing. Curr Med Chem 19: 90-97, 2012.

9. Ben-Yosef Y, Lahat N, Shapiro S, Bitterman H and Miller A: Regulation of endothelial matrix metalloproteinase-2 by hypoxia/reoxygenation. Circ Res 90: 784-791, 2002.

10. Horiuchi K, Amizuka N, Takeshita S, et al: Identification and characterization of a novel protein, periostin, with restricted expression to periosteum and periodontal ligament and increased expression by transforming growth factor beta. J Bone Miner Res 14: 1239-1249, 1999.

11. Zhou HM, Wang J, Elliott C, Wen W, Hamilton DW and Conway SJ: Spatiotemporal expression of periostin during skin development and incisional wound healing: lessons for human fibrotic scar formation. J Cell Commun Signal 4: 99-107, 2010.

12. Kyutoku M, Taniyama Y, Katsuragi N, et al: Role of periostin in cancer progression and metastasis: Inhibition of breast cancer progression and metastasis by anti-periostin antibody in a murine model. Int J Mol Med 28: 181-186, 2011.

13. Wang Q, Nie FF, Zhao X and Qin ZL: The expression of periostin in hyperplasic scars and the relations to TGF-betal and its receptors. Zhonghua Zheng Xing Wai Ke Za Zhi 23: 229-232, 2007 (In Chinese).

14. Liu C, Song ZH and Qin ZL: Construction of periostin shRNA vectors and their effects on the expression of periostin in fibroblasts. Beijing Da Xue Xue Bao 42: 503-508, 2010.

15. Kong D, Park EJ, Stephen AG, et al: Echinomycin, a smallmolecule inhibitor of hypoxia-inducible factor-1 DNA-binding activity. Cancer Res 65: 9047-9055, 2005.

16. Alster TS and Tanzi EL: Hypertrophic scars and keloids: etiology and management. Am J Clin Dermatol 4: 235-243, 2003.

17. Song J, Xu H, Lu Q, et al: Madecassoside suppresses migration of fibroblasts from keloids: involvement of p38 kinase and PI3K signaling pathways. Burns 38: 677-684, 2012. 
18. Syed F, Sherris D, Paus R, Varmeh S, Pandolfi PP and Bayat A: Keloid disease can be inhibited by antagonizing excessive mTOR signaling with a novel dual TORC1/2 inhibitor. Am J Pathol 181: $1642-1658,2012$

19. Gillan L, Matei D, Fishman DA, Gerbin CS, Karlan BY and Chang DD: Periostin secreted by epithelial ovarian carcinoma is a ligand for alpha(V)beta(3) and alpha(V)beta(5) integrins and promotes cell motility. Cancer Res 62: 5358-5364, 2002.

20. Chung KC, Disa JJ, Gosain AK, Kinney BM and Rubin JP Plastic Surgery. 1st edition. Elsevier, New York, 2009.

21. Lokmic Z, Musyoka J, Hewitson TD and Darby IA: Hypoxia and hypoxia signaling in tissue repair and fibrosis. Int Rev Cell Mol Biol 296: 139-185, 2012.

22. Kischer CW: The microvessels in hypertrophic scars, keloids and related lesions: a review. J Submicrosc Cytol Pathol 24: 281-296, 1992.

23. Kischer CW, Thies AC and Chvapil M: Perivascular myofibroblasts and microvascular occlusion in hypertrophic scars and keloids. Hum Pathol 13: 819-824, 1982.

24. Watanabe T, Yasue A, Fujihara S and Tanaka E: PERIOSTIN regulates MMP-2 expression via the $\alpha v \beta 3$ integrin/ERK pathway in human periodontal ligament cells. Arch Oral Biol 57: 52-59, 2012.

25. Li P, Oparil S, Feng W and Chen YF: Hypoxia-responsive growth factors upregulate periostin and osteopontin expression via distinct signaling pathways in rat pulmonary arterial smooth muscle cells. J Appl Physiol (1985) 97: 1550-1558; discussion 1549,2004

26. Deschene K, Céleste C, Boerboom D and Theoret CL: Hypoxia regulates the expression of extracellular matrix associated proteins in equine dermal fibroblasts via HIF1. J Dermatol Sci 65: 12-18, 2012.

27. Vincent AS, Phan TT, Mukhopadhyay A, Lim HY, Halliwell B and Wong KP: Human skin keloid fibroblasts display bioenergetics of cancer cells. J Invest Dermatol 128: 702-709, 2008.

28. Mucaj V, Shay JE and Simon MC: Effects of hypoxia and HIFs on cancer metabolism. Int J Hematol 95: 464-470, 2012.

29. Chen J, Zeng B, Yao H and Xu J: The effect of TLR4/7 on the TGF- $\beta$-induced Smad signal transduction pathway in human keloid. Burns 39: 465-472, 2013

30. Bran GM, Goessler UR, Schardt C, Hormann K, Riedel F and Sadick H: Effect of the abrogation of TGF- $\beta 1$ by antisense oligonucleotides on the expression of TGF- $\beta$-isoforms and their receptors I and II in isolated fibroblasts from keloid scars. Int J Mol Med 25: 915-921, 2010.
31. Wu CS, Wu PH, Fang AH and Lan CC: FK506 inhibits the enhancing effects of transforming growth factor (TGF)- $\beta 1$ on collagen expression and TGF- $\beta /$ Smad signalling in keloid fibroblasts: implication for new therapeutic approach. Br J Dermatol 167: 532-541,2012.

32. Bettinger DA, Yager DR, Diegelmann RF and Cohen IK: The effect of TGF-beta on keloid fibroblast proliferation and collagen synthesis. Plast Reconstr Surg 98: 827-833, 1996.

33. Lindsley A, Li W, Wang J, Maeda N, Rogers R and Conway SJ: Comparison of the four mouse fasciclin-containing genes expression patterns during valvuloseptal morphogenesis. Gene Expr Patterns 5: 593-600, 2005.

34. Naik PK, Bozyk PD, Bentley JK, et al: Periostin promotes fibrosis and predicts progression in patients with idiopathic pulmonary fibrosis. Am J Physiol Lung Cell Mol Physiol 303: L1046-L1056, 2012.

35. Huang L, Wong YP, Cai YJ, Lung I, Leung CS and Burd A: Low-dose 5-fluorouracil induces cell cycle G2 arrest and apoptosis in keloid fibroblasts. Br J Dermatol 163: 1181-1185, 2010.

36. Ouyang G,Liu M, Ruan K, Song G, Mao Y and Bao S: Upregulated expression of periostin by hypoxia in non-small-cell lung cancer cells promotes cell survival via the Akt/PKB pathway. Cancer Lett 281: 213-219, 2009.

37. Greijer AE and van der Wall E: The role of hypoxia inducible factor 1 (HIF-1) in hypoxia induced apoptosis. J Clin Pathol 57: 1009-1014, 2004.

38. Bao S, Ouyang G, Bai X, et al: Periostin potently promotes metastatic growth of colon cancer by augmenting cell survival via the Akt/PKB pathway. Cancer Cell 5: 329-339, 2004.

39. Barrientos S, Stojadinovic O, Golinko MS, Brem H and TomicCanic M: Growth factors and cytokines in wound healing. Wound Repair Regen 16: 585-601, 2008.

40. Hou JJ, Nie FF, Li BL, et al: The expressions of periostin and the related factors during healing process of full-thickness cutaneous wound in rat. Zhongguo Wei Zhong Bing Ji Jiu Yi Xue 24: 334-337, 2012 (In Chinese).

41. Elliott CG, Wang J, Guo X, et al: Periostin modulates myofibroblast differentiation during full-thickness cutaneous wound repair. J Cell Sci 125: 121-132, 2012.

42. Shimazaki M, Nakamura K, Kii I, et al: Periostin is essential for cardiac healing after acute myocardial infarction. J Exp Med 205: 295-303, 2008. 